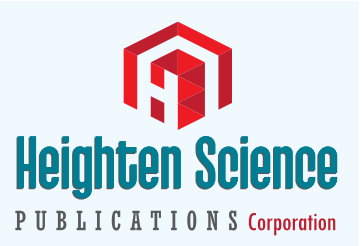

ISSN

2639-9954

\title{
Peculiarities of photon emisson of whole non-diluted human blood obtained from healthy donors and patients with some diseases
}

\author{
Kirill N Novikov ${ }^{1 *}$, Vladimir L Voeikov ${ }^{1}$, Ekaterina V Buravleva ${ }^{1}$ \\ and Nadezhda G Berdnikova ${ }^{2}$
}

${ }^{1}$ Faculty of Biology, MV Lomonosov Moscow University, Moscow, Russia

${ }^{2}$ I.M.Sechenov first Moscow Medical State University, Moscow, Russia

*Address for Correspondence: Novikov KN Faculty of Biology, MV Lomonosov Moscow University, Moscow, Russia, Tel: +7 (495) 93912 68; Email: kirniknov@yandex.ru

Submitted: 24 December 2018 Approved: 28 December 2018 Published: 31 December 2018

Copyright: ๑ 2018 Novikov KN, et al. This is an open access article distributed under the Creative Commons Attribution License, which permits unrestricted use, distribution, and reproduction in any medium, provided the original work is properly cited

Keywords: Whole non-diluted blood; Chemiluminescence; Photon emission; Luminol; Lucigenin; Respiratory burst; Reactive oxygen species; Non-radiative energy transfer; Angina pectoris; Chronic obstructive pulmonary disease

Check for updates

\section{Abstract}

Blood plays an important role in oxygen absorption and its transfer to organs and tissues in vertebrates, as well as in a number of invertebrate species. Numerous interactions between cellular and non-cellular blood components constantly occur. A special role in these interactions belongs to erythrocytes and leukocytes, between which oxygen is constantly exchanged and activated, which we showed directly in whole blood. Blood is a liquid tissue, which is a complex cooperative system and has many inherent functions and the most important one is the ability to maintain the homeostasis of the body. Our experience has shown that despite its high optical density, undiluted blood of humans and animals can be a source of radiation due to the transformation of the energy of electron-excited (EEE) states and secondary processes occurring in the whole blood system. Parameters of this radiation - ultra-weak photons emission (UWPE) from blood - depend upon its physiological properties and reflect the physiological state of a donor. Analysis of UWPE from non-diluted blood is a simple and sensitive method that allows to monitor the course of treatment of a patient. In spite of high opacity of nondiluted blood it may be a strong source of UWPE both in the presence and absence of UWPE enhancers. Analysis of patterns of UWPE from blood reveals its highly non-linear, stable non-equilibrium and cooperative properties. Characteristic of a living system.

\section{Introduction}

Blood is the most important function in the absorption, transfer and transfer to organs and tissues of oxygen in most species of vertebrates, as well as in a number of invertebrate species. In itself, numerous interactions between cellular and noncellular components constantly occur. A special role in these interactions belongs to erythrocytes and leukocytes, between which oxygen is constantly exchanged and activated, which we showed directly in whole blood. Blood is a liquid tissue, which is a complex cooperative system, the most important of its properties is the ability to maintain the homeostasis of the body. Directly reflect the significance of reactive oxygen species (ROS) in the formation various regulatory functions in the blood. Our experience has shown that despite its high optical density, non-diluted blood of humans and animals can be a source of radiation due to the transformation of EEE states and resulting from secondary processes occurring in the whole blood system. Parameters of this radiation - UWPE from blood depend upon its physiological properties and reflect the physiological state of a donor. Analysis of UWPE from non-diluted blood is a simple and sensitive method allowing to monitor the course of treatment of a patient. In spite of high opacity of non-diluted blood it may be a strong source of UWPE both in the presence and absence of UWPE enhancers. Patterns of UWPE from blood argue 
that ROS are continuously generated in it, in particular those that are registered in the presence of the radiation activator lucigenin (LC). Analysis of patterns of UWPE from blood reveals its highly non-linear, stable non-equilibrium and cooperative properties, characteristic of a living system $[1,3]$.

\section{Materials and Methods}

Blood of healthy donors and patients with different diseases was obtained by venous puncture and was stabilized by heparine. Blood was obtained between 9.00 and $10.00 \mathrm{a}$. m. and used exactly 3 hours after extravasation. During this period it was kept in plastic tubes at $20-25^{\circ} \mathrm{C}$.

The study was approved by the Hospital Medical Ethical Committee (Moscow City Clinical Hospital N23 «Medsantrud»), and all the patients gave informed consent.

\section{The method for recording chemiluminescence (CL) - UWPE}

Using the method of CL, reactive oxygen species (ROS) status in non-diluted whole blood of patients and healthy donors was investigated. UWPE was registered using two different types of single photon counters. In first experiments one was the liquid scintillation counter Mark-II (Nuclear-Chicago, USA), equipped with photomultipliers EMI 9750QB/I. It was used in the mode of single photon counting (coincidences circuit off) at a maximal gain. The measurements were performed at room temperature (19$2 \mathrm{I}^{\circ} \mathrm{C}$ ). Dark counts varied in the range of $20-40$ counts/sec.

Standard borosylicate glass vials, or $1 \mathrm{ml}$ Eppendorf polyethylene test tubes were used as blood containers. Test tubes were fixed in empty glass vials always in one and the same position. Vials and test tubes having negligibly low background count-rate and short decay time of own luminescence after insertion into the counting chamber were selected. Parameters of CL from blood did not depend upon the material of vials. Blood (0.2-I.0 ml) was added to a vial or a test tube, its count-rate/0.2 min was registered for 2-3 $\mathrm{min}$, and luminol (LM) (Sigma) was added to a blood sample to a final concentration of $10^{-4} \mathrm{M}$ from stock solution $\left(10^{-1} \mathrm{M}\right)$ in analytical grade dimethyl sulfoxide.

The second single photon counter was chemiluminometer "Biotoks-7a" (Russia) (Figure 1).

The device is equipped with the horizontal photoelectronic multiplier (PM) working as a single photon counter (9750QB / 1 PM, EMI Electronics LTD, Middlesex, UK), the spectral sensitivity region is $380-710 \mathrm{~nm}$ with a maximum in the blue-green region at $420-500 \mathrm{~nm}$. Aliquots of blood $(100 \mu \mathrm{l})$ was placed in the Eppendorf type test tube, then lucigenin (LC) was added (final concentration $-10^{-4} \mathrm{M}$ ) and CL kinetics were measured for 300 seconds, and photon counting was done every second. The data are presented as photon counts per second and light sums which are integrals

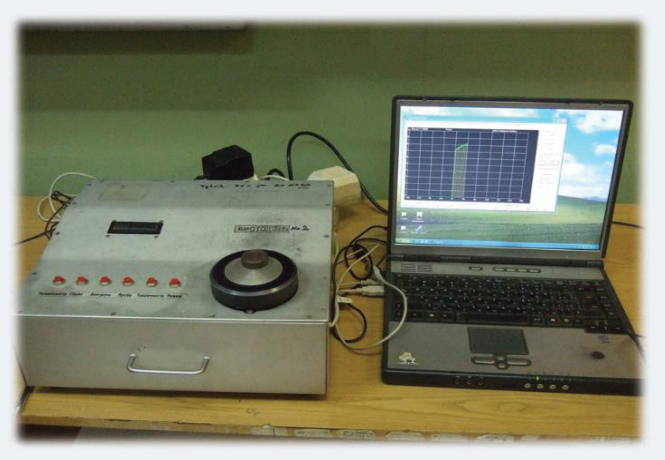

Figure 1: Equipment for the registration of non-diluted whole blood CL (chemiluminometer "Biotox-7A"). 
of kinetic CL curves of 300 seconds. The results were processed in the Statistics and Excel programs, reliability of differences of the experimental and control values was determined according to the criteria of Student, Manni-Whitney and Wilcoxon.

\section{Results and Discussion}

\section{Properties of UWPE of whole non-diluted blood of healthy donors}

UWPE of biological objects is considered to arise as an immediate result of relaxation of electron xcited states (EES) of products of free radical oxidative reactions with the participation of ROS [2]. There are a lot of studies of UWPE accompanying respiratory burst (RB) induced in neutrophil suspensions or highly diluted blood preparations. Non-diluted blood is not used for these studies, because it is supposed that hemoglobin and other chromofores should completely quench UWPE. However, we observed CL from non-diluted human blood in the absence and presence of CL amplifiers (luminol and especially lucigenin) even in a resting state. We obtained the dependence of LM and LC amplified CL of a healthy donor's blood in the absence of RB stimulants upon time after blood has been (Figure 2).

Major energy source for ROS generation in whole blood are reactions of oneelectron oxygen reduction performed principally by white blood cells; at that red blood cells (RBC) serve as oxygen source for these reactions. Hemoglobin ( $\mathrm{Hb}$ ) packed in RBC providing opacity to blood actually did not quench CL from it. Luminescence practically disappeared when free Hb was added to blood. Thus UWPE from blood may result from the secondary emission due to energy transfer in this live cooperative system (Figure 3).

Response of blood of a healthy donor (Figure 4) and of an infarction patient (Figure 5) on withdrawal of its portions and evolution of the property during and evolution of this property during blood storage indicates of highly non-linear and cooperative properties of this living system.

\section{Role of UWPE of whole non-diluted blood of an infarction and stable angina pectoris patients}

We studied the LM-dependent photon emission from non-diluted blood of stable angina pectoris patient before and after low-level intravenous laser therapy (Figure 6). Blood was taken from patients just after their admission to the hospital, and $24 \mathrm{hr}$. after the 3-d, 7(8)-th, and laser session just before the current treatment. Note that unlike a healthy donors' blood addition of LM to it is followed with animmediate increase in UWPE [4].

Patient "M.", 63 years old, male, had the and post - following diagnosis on admission: myocardial ischemia, stenocardia of tension (functional class III), atherosclerotic
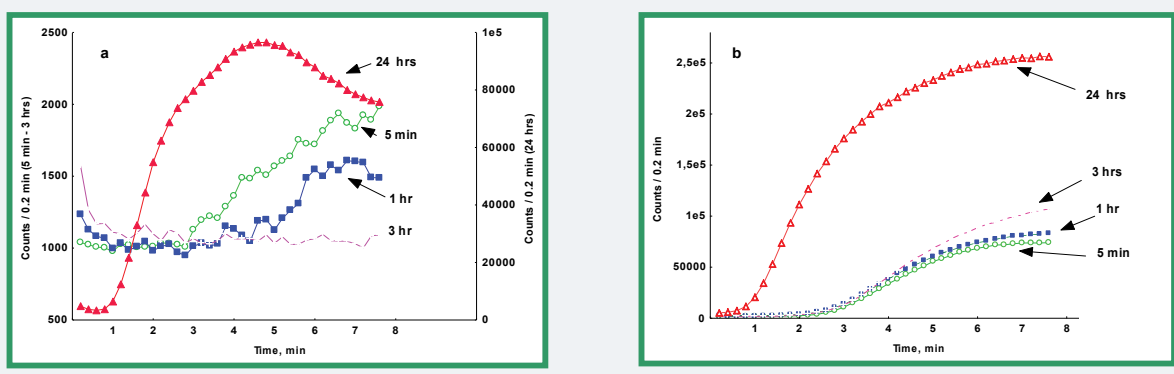

Figure 2: Photon emission changes in non-diluted blood $(0.2 \mathrm{ml})$ supplemented with $L M(a)$ or LC (b) in relation to time of blood storage. LM - a probe for multiple forms of ROS, predominantly H2O2 and CIO-generation; LC - a probe for 02 - generation. Aliquots $(0.2 \mathrm{ml})$ for measurements were taken from this sample at time moments marked by inscriptions at each curve. Note, that the curves for $5 \mathrm{~min} ., 1 \mathrm{hr}$, and 3 hours in (a) apply to the left ordinate and the curve for 24 hours - to the right ordinate. Liquid scintillation counter Mark-II was used (Mark-II was used). 


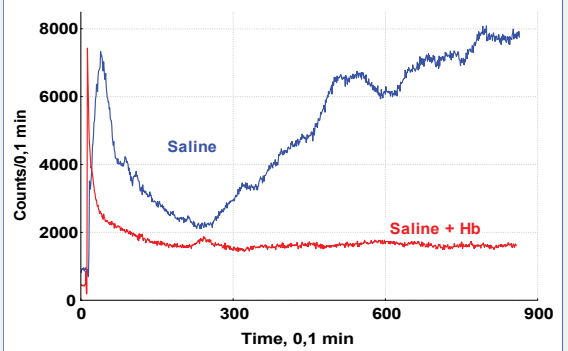

Figure 3: LC-dependent UWPE from blood diluted 1:1 with physiological saline (blue curve) or physiological saline to which human hemoglobin was added ait a concentration of $2 \mathrm{mg} / \mathrm{ml}$ (red curve). Mark-II was used.

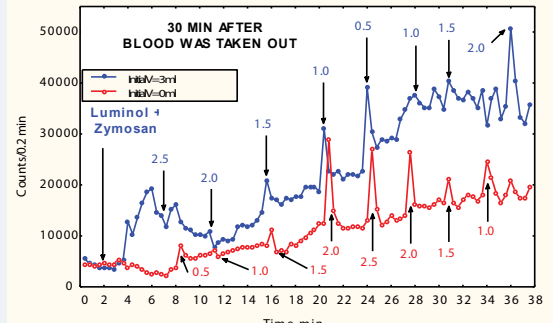

Time, $\min$

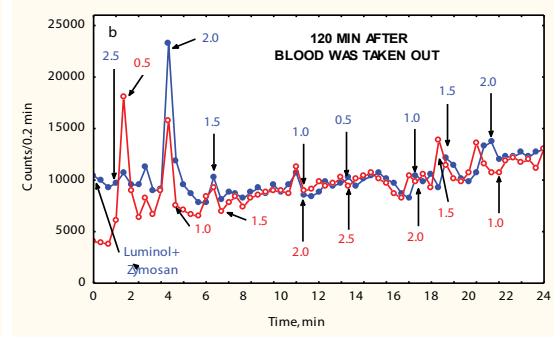

Time, $\min$

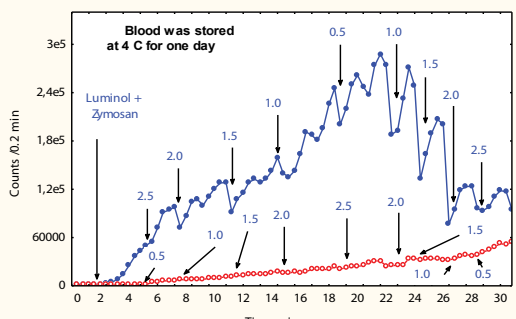

Figure 4: $3 \mathrm{ml}$ of a healthy donor's blood was added to a vial and PE from it (blue line) was registered in turn with an empty vial. At indicated time points aliquotes of blood were transferred to an empty vial (red line). Mark-II was used.
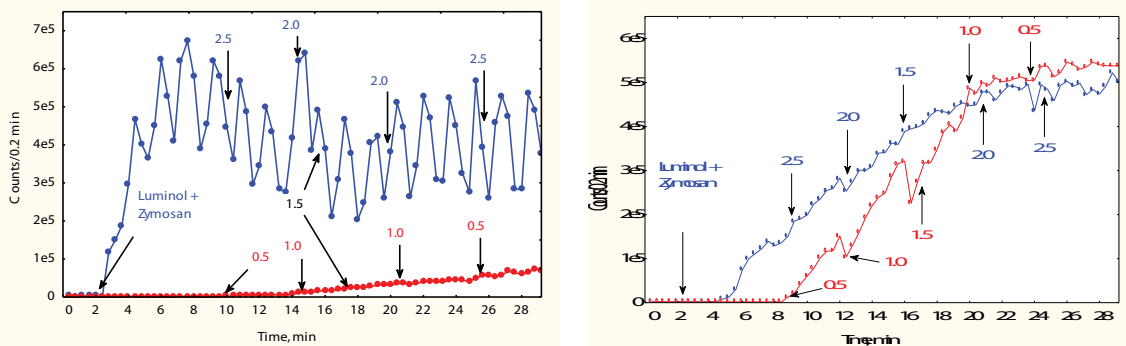

Figure 5: Response of blood of a patient on withdrawal of its portions changes in the course of the patient recovery (left fig. - shortly after infarction; right fig. - after 4 days of treatment). Mark-ll was used.

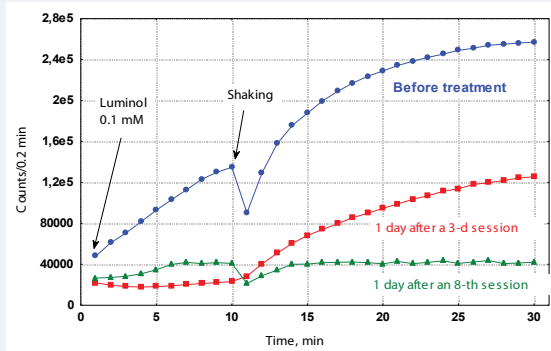

Figure 6: Luminol-dependent photon emission from non-diluted blood of stable angina pectoris patient "M." before (blue curve) and after low-level intravenous laser therapy (3-d laser session - red curve and 8-th laser session green curve): laser treatment session: $30 \mathrm{~min}$. exposure to $\mathrm{l}=633 \mathrm{~nm}$, output power $-1 \mathrm{~mW}$, delivered into elbow vein through a waveguide. Mark-II was used. 
infarction (1987) cardiosclerosis, chronic aneurysm of the heart, blood flow insufficiency stage 1. After 8 sessions of low level laser therapy combined with chemotherapy (nitrates, beta-adrenergic antagonists, aspirin) the patient was feeling well. Just after his admission to the hospital LM addition to blood induced fast and very intensive growth of PE intensity (Figure 6, blue curve). Mild shaking of a sample was followed with a new acceleration of CL intensity growth. 24 hours following the 3-d laser session CL of his blood dropped already to values characteristic for healthy donors' blood, though after a sample was shaken CL intensity began to grow. After the 8-th laser session addition of LM to blood resulted in a moderate elevation of photon emission, but sample shaking did not cause additional increase in CL. Our further studies on LMdependent CL in the blood of angina pectoris patients during laser therapy have shown that it is possible not only to diagnose the improvement of patients, but also to warn about the timely termination of laser therapy sessions in the event of an increase in the intensity of blood CL [4].

\section{Chronic obstructive pulmonary disease (COPD) and patients' blood UMPE}

COPD is characterized by not fully reversible airflow limitation (bronchial obstruction), which usually progresses steadily and is caused by an inflammatory reaction of lung tissue on the impact of pathogenic particles or gases [5]. COPD is an inflammatory disease associated with ROS production. ROS are highly chemically reactive and need to be controlled to prevent damage [6,7]. The system of control is generally known as an antioxidant system. In this exploration the effect of Hypoxen ${ }^{\circledR}$ [8], treatment on ROS production, the formation of which is recorded in dynamics CL, in patients' blood was studied (Figure 7). Recorded in blood RB in the presence of LM and zymosan (ZM) (activator of RB) and LC that determines the rate of $\mathrm{O}_{2}{ }^{-}$generation.

Changes in the dynamics of the average values of the intensity of whole blood CL in 30 patients with COPD before (2) and after (3) therapy by Hypoxen ${ }^{\circledR}$ and during acute attack (1). Hypoxen ${ }^{\circledR}$ which is the new promising antioxidant and antihypoxant that demonstrates remarkable ROS protective effect in patients with COPD and appears to have very few contradictions. Chemiluminometer "Biotoks-7a" was used.

The aim of the current study was to compare the level of ROS in the whole undiluted blood of 3 groups of volunteers: patients with COPD before treatment with Hypoxen ${ }^{\circledR}$, patients with COPD after treatment with Hypoxen ${ }^{\circledR}$, and patients suffering from acute attack. A statistically significant decrease in ROS contents in blood was observed after Hypoxen ${ }^{\circledR}$ treatment of COPD [8]. Thus in all the patients with COPD in remission phase with Hypoxen ${ }^{\circledR}$ appointment, LM-CL with ZM and LC-enhanced CL (LC-CL) decreased after the treatment. Parameters of CL xcited states (EES) of products of free radical oxidative reactions with the participation of ROS [2]. There are a lot of studies of UWPE accompanying respiratory burst (RB) induced in neutrophil suspensions or highly diluted blood preparations. Non-diluted blood is not used for these studies, because it

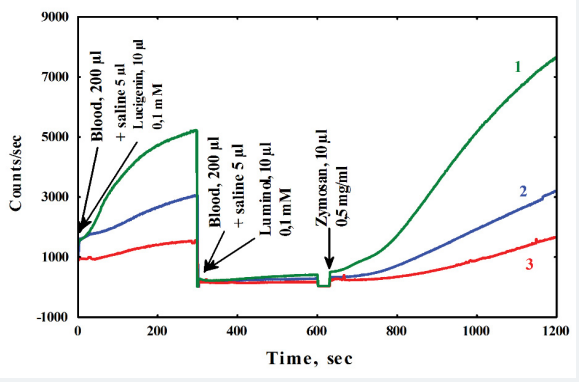

Figure 7: Changes in the dynamics of the average values of the intensity of whole blood CL in 30 patients with COPD before (2) and after (3) therapy by Hypoxen ${ }^{\circledR}$ and during acute attack (1). Hypoxen ${ }^{\circledR}$ which is the new promising antioxidant and antihypoxant that demonstrates remarkable ROS protective effect in patients with COPD and appears to have very few contradictions. Chemiluminometer "Biotoks-7a" was used. 
is supposed that hemoglobin and other chromofores should completely quench UWPE. However, we observed CL from non-diluted human blood in the absence and presence of CL amplifiers (luminol and especially lucigenin) even in a resting state. We obtained the dependence of LM and lucigenin (LC) amplified CL of a healthy donor's blood in the upon them depended on blood state. Using the discriminator analysis we found the most important time points in the kinetic curves of CL for classification patients into groups (e.g., COPD patients before and after treatment with Hypoxen ${ }^{\circledR}$ ) [9].

\section{Conclusion}

In spite of high opacity of non-diluted blood it may be a strong source of photon emission both in the presence and absence of PE enhancers. Parameters of PE from blood depend upon its physiological properties and reflect the physiological state of a donor. Analysis of PE from non- diluted blood is a simple and sensitive method allowing to monitor the course of treatment of a patient. Patterns of PE from blood argue that ROS are continuously generated in it, in particular those that are registered in the presence of LC. Analysis of patterns of UWPE from blood reveals its highly stable non-equilibrium and cooperative properties, characteristic of a living system $[1,3]$. A seemingly paradoxical effect of first laser sessions - a decrease of blood LMCL, rather than its elevation after blood irradiation needs some explanation. First, it should be stressed, that blood analysis was performed 24 hours after intravenous blood irradiation. It means that we observed some generalized effects of the preceding session, rather than a direct effect of laser irradiation of blood. Then the question arises for the reasons of high and low LM-CL from non- diluted blood. It is generally supposed that that PE from living cells and tissues is an immediate outcome of relaxation of excited states arising from free radical oxidative reactions [10-12]. However, if this concept may be true for more or less severely perturbed systems (from simple blood dilution [13-15] to homogenates $[12,16]$ its extrapolation to non-diluted blood, that is blood in its maximally native ex vivo state is questionable. Hypoxen ${ }^{\circledR}$ treatment of COPD patients resulted in significant alternation of LM-ZM-dependent and LC-dependent CL of whole blood of patients. This is consistent with the claimed strong antioxidant activity of Hypoxen ${ }^{\circledR}$ [9].

\section{References}

1. Voeikov VL, Novikov CN, Vilenskaya ND. Low-level chemiluminescent analysis of nondiluted human blood reveals its dynamic system properties. J Biomed Opt. 1999; 4: 54-60. Ref.: https://goo.gl/UL1Mw8

2. Voeikov VL. Reactive Oxygen Species (ROS): Pathogens or Sources of Vital Energy? Part 1. ROS in Normal and Pathologic Physiology of Living Systems. J Altern Complement Med. 2006' 12: 111-118. Ref.: https://goo.gl/119vXP

3. Voeikov VL, Asfaramov R, Bouravleva EV, Novikov CN, Vilenskaya ND. Vilenskaya N.D. Biophoton research in blood reveals its holistic properties. Indian J. Exp. Biol., 2003; 43: 473-482. Ref.: https://goo.gl/VQhwD3

4. Voeikov VL, Novikov CN, Siuch NI, Alterations in Luminol-enhanced CL from nondiluted whole blood in the course of low-level laser therapy of angina pectoris patients. In: Cohn, G.E, Soper, S.A., eds. Ultrasensitive Biochemical Diagnostics II, SPIE Proc. 1997; 2985: 286-294. Ref.: https://goo.gl/wRtsFZ

5. MacNee W, Tuder RM. New Paradigms in the Pathogenesis of Chronic Obstructive Pulmonary Disease. Proceedings of the American Thoracic. Society. 2009; 6: 527-531. Ref.: https://goo.gl/z7NVkw

6. Skyba P, Kluchova Z, Joppa P, Petrasova D, Tkacova R. Nutritional status in relation to respiratory impairment and systemic inflammation in patients with acute exacerbations of COPD. Med Sci Monit. 2009; 15: 528-533. Ref.: https://goo.gl/AbJRQD

7. Cavalcante AGM, de Bruin PFC. The role of oxidative stress in COPD: current concepts and perspectives. J Bras Pneumol. 2009; 35: 1227-1237. Ref.: https://goo.gl/EGueDW

8. Smirnov VS, Kuzmich MK. Hypoxen. St. Petersburg-Moscow, 2001 (in Russian).

9. Novikov KN, Berdnikova NG, Novikov AK, Lyusina OY, Muhitova OG, et al. Changes in chemiluminescence of whole blood of COPD patients treated with Hypoxen ${ }^{\circledR}$ and effects of $\mathrm{C}_{60}$ fullerenes on blood chemiluminescence. Med Sci Monit. 2012; 18: 76-83. Ref.: https://goo.gl/twDphn 
10. Zhuravlev AI. Ed. Biochemiluminescence, USSR Acad. of Sci. and Moscow Soc., Nature. 1983; 58: 210-222.

11. Vladimirov YA. Sherstnev MP. Chemiluminescence of living cells, Moscow, VINITI Publ. House, Series "Biopysics", 1989; 24.

12. Cadenas E. Biological chemiluminescence, Photochem. Photobiol. 1984; 40: 823-830, 1984. Ref.: https://goo.gl/UBE3GZ

13. Bochev BG1, Magrisso MJ, Bochev PG, Markova VI, Alexandrova ML. Dependence of whole blood luminol chemiluminescence on PMNL and RBC count. J Biochem Biophys Methods. 1993; 27: 301309. Ref.: https://goo.gl/VweNJK

14. Kaever V1, Robitzsch JT, Stangel W, Schleinkofer L, Resch K. Simultaneous detection of whole blood chemiluminescence in microtitre plates. Eur J Clin Chem Clin Biochem. 1992; 30: 209-216. Ref.: https://goo.gl/wJLC4x

15. Descamps-Latscha B, Nguyen AT, Golub RM, Feuillet-Fieux MN. Chemiluminescence in microamounts of whole blood for investigation of the human phagocyte oxidative metabolism function. Ann Immunol (Paris). 1982; 3: 349-364. Ref.: https://goo.gl/LSX3mS

16. Murphy ME, Sies $\mathrm{H}$. Visible-range low-level chemiluminescence in biological systems. Methods Enzymol. 1990; 186: 595-610. Ref.: https://goo.gl/a6qMCr 